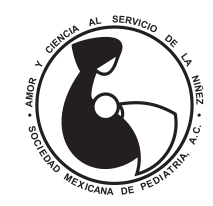

\title{
Laparoscopía en el diagnóstico de síndrome de persistencia del conducto de Müller en un escolar con escroto vacío
}

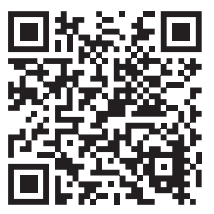

\author{
Alfonso Galván-Montaño,‡ José Alfonso Maya-Barrios, ${ }^{\S}$ Sara Parraguirre-Martínez,॥ \\ Marco Antonio Jiménez-Ferreira," Rubí Azeneth Baca-Patiño,\| Silvia García-Moreno ${ }^{\Uparrow, *}$ \\ ‡ Cirugía Pediátrica; ${ }^{\S}$ División de Pediatría; "| División de Anatomía Patológica; ` División de Investigación Clínica. Hospital \\ General "Dr. Manuel Gea González", Ciudad de México, México.
}

\begin{abstract}
RESUMEN
Introducción: La laparoscopía se ha utilizado ampliamente para diagnosticar y en el tratamiento de testículos no palpables. Hasta nuestro conocimiento, no hay reportes de casos con síndrome de persistencia del conducto de Müller (SPCM) diagnosticado por laparoscopía. Caso clínico: Niño de seis años con criptorquidia izquierda y bolsa escrotal vacía, el testículo derecho en bolsa escrotal. Por ultrasonido, el testículo derecho fue normal, pero no se detectó el testículo izquierdo. En la laparoscopía se encontraron estructuras que correspondieron a útero, trompa y ligamento redondo. El estudio histopatológico reportó útero (miometrio y endometrio), salpinge y restos de corteza suprarrenal. Conclusiones: EI SPCM debe considerarse como una causa en niños con escroto vacío, siendo la laparoscopía una buena alternativa diagnóstica y terapéutica.
\end{abstract}

Palabras clave: Conducto de Müller persistente, criptorquidia, laparoscopía, trastornos del desarrollo sexual.

\section{INTRODUCCIÓN}

La laparoscopía se ha utilizado cuando no se palpan los testículos desde $1976 .{ }^{1}$ El testículo no descendido es una de las anomalías congénitas más comunes,

\begin{abstract}
Introduction: Laparoscopy has been widely used to diagnose and treat non-palpable testes. To our knowledge, there are no reports of cases with persistent Müllerian duct syndrome (PMDS) diagnosed by laparoscopy. Clinical case: Six year-old boy with left cryptorchidism and empty scrotal sac; right testicle was in his scrotal sac. By ultrasound, the right testicle was normal, but the left testicle was not detected. At laparoscopy, structures corresponding to the uterus, tube and round ligament were found. Histopathological study reported uterus (myometrium and endometrium), salpinge and remains of adrenal cortex. Conclusions: PMDS should be considered as a cause of empty scrotum in children, and laparoscopy as a good diagnostic and therapeutic alternative.
\end{abstract}

Keywords: Persistent Müllerian duct syndrome, cryptorchidism, laparoscopy, sexual development disorders.

que afecta hasta $4 \%$ de los recién nacidos de término y hasta $45 \%$ de los prematuros. De éstos, $20 \%$ se reportan como testículo no palpable (escroto vacío) e incluye varias condiciones patológicas como los testículos intraabdominales, testículos en tercio su-

\footnotetext{
* Correspondencia: SGM, silviagarciamoreno1901@gmail.com

Conflicto de intereses: Los autores declaran que no tienen.

Citar como: Galván-Montaño A, Maya-Barrios JA, Parraguirre-Martínez S, Jiménez-Ferreira MA, Baca-Patiño RA, García-Moreno S. Laparoscopía en el diagnóstico de síndrome de persistencia del conducto de Müller en un escolar con escroto vacío. Rev Mex Pediatr 2020; 87(3):102-105. doi: 10.35366/94840

[Laparoscopy in the diagnosis of persistent Müllerian duct syndrome in a scholar with empty scrotum]
} 
perior de canal inguinal, atrofia testicular y testículo evanescente. $^{2}$

Cuando en la exploración física inicial, el testículo no es palpable, se puede realizar ecografía, tomografía axial o resonancia magnética, en las cuales su exactitud es de hasta $44 \% .^{3-5}$ Mientras que la laparoscopía no sólo tiene mayor sensibilidad y especificidad, ${ }^{6-9}$ sino que permite determinar la viabilidad testicular, si existe atrofia o ausencia de la gónada, o bien, la presencia de remanentes testiculares. Además, este procedimiento es terapéutico. ${ }^{5,9}$

El síndrome de persistencia del conducto de Müller (SPCM) fue descrito por primera vez por Nilson en 1939. Posteriormente, alrededor de 150 casos se han reportado en la literatura. Es una enfermedad rara que ocurre en hombres con un fenotipo completa o genotípicamente normal, y se caracteriza por la presencia de estructuras del conducto de Müller. El paciente con SPCM puede tener criptorquidia unilateral o bilateral. Dado que los pacientes son fenotípicamente mascu-

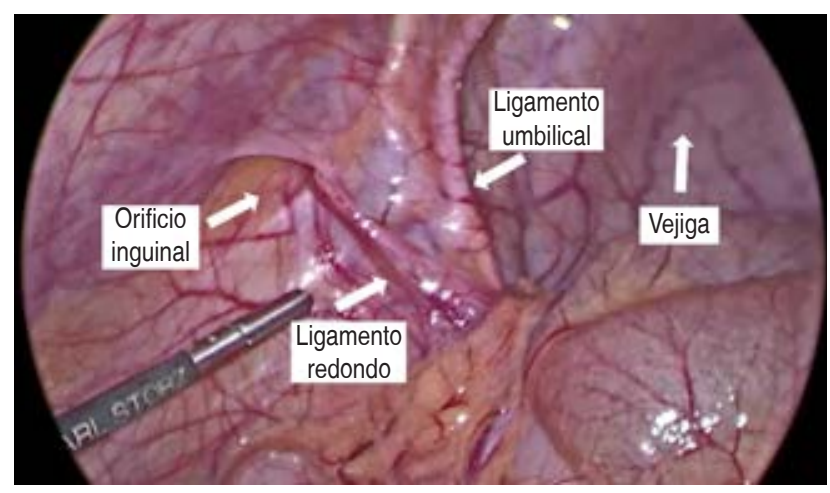

Figura 1: Se observa el orificio inguinal permeable izquierdo, ligamento redondo, ligamento umbilical y la vejiga.

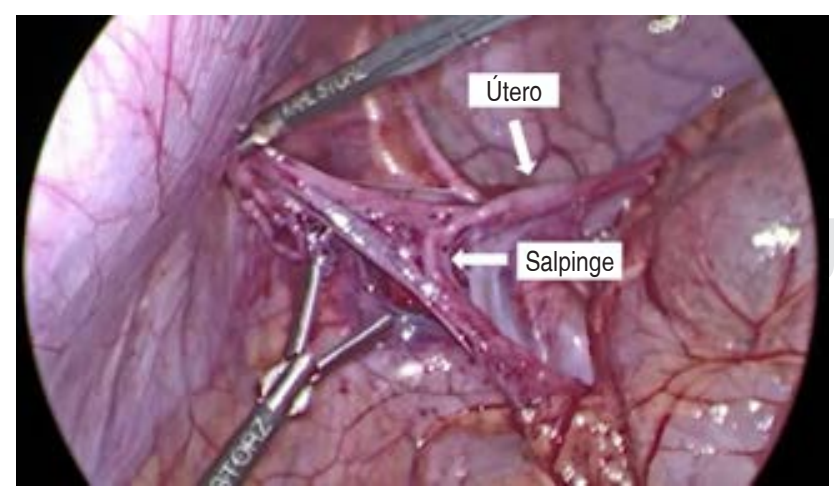

Figura 2: Se identifica útero y salpinge izquierda.

Tabla 1: Características y porcentaje de los casos del
síndrome persistente del conducto de Müller.

linos, por lo general no se sospecha este diagnóstico hasta que se lleva a cabo cirugía para criptorquidia o al reparar una hernia inguinal. ${ }^{10}$

El objetivo de presentar el caso clínico es reconocer la importancia de la laparoscopía en el diagnóstico de SPCM.

\section{PRESENTACIÓN DEL CASO}

Niño de seis años de edad referido a nuestro hospital por criptorquidia izquierda. Tenía antecedente de trastorno del espectro autista, así como déficit de atención e hiperactividad, tratado con metilfenidato 10 meses previos a su ingreso.

A la exploración física se encontró bolsa escrotal izquierda vacía, sin palparse el testículo en escroto ni en canal inguinal. El testículo derecho se localizó en su bolsa escrotal, el cual tenía tamaño normal de acuerdo a su edad. El pene de características normales.

Por ultrasonido se observó testículo derecho con bordes regulares, dimensiones de $18.0 \times 7.6 \times 10.0$ $\mathrm{mm}$; epidídimo derecho en su porción cefálica con dimensiones de $6.4 \times 4.7 \mathrm{~mm}$ y en su porción caudal con dimensiones de $4.7 \times 3.1 \mathrm{~mm}$, ecografía homogénea, hipoecoica, sin alteraciones. En la bolsa escrotal izquierda no se observó alguna imagen compatible con testículo, por lo que se exploró el canal inguinal hasta su tercio proximal, pero tampoco se encontró.

Con el diagnóstico de escroto vacío se procedió a realizar laparoscopía diagnóstica que detectó peque- 

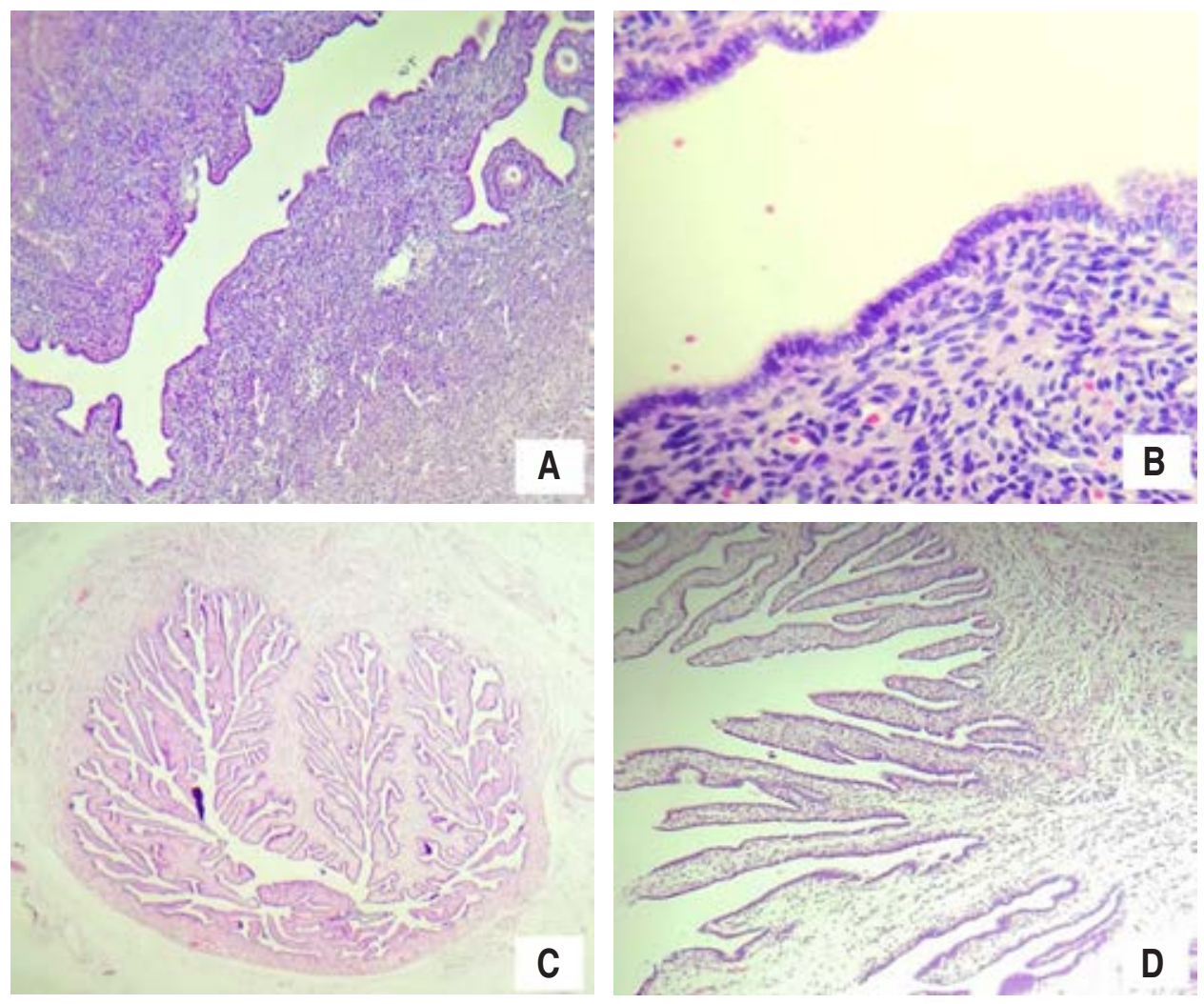

Figura 3:

A) Foto panorámica de estructuras correspondientes a útero (endometrio, miometrio). B) Acercamiento de la mucosa endometrial que muestra estroma compacto revestido de epitelio cilíndrico. C) Cortes histológicos de salpinge caracterizada por pliegues que se proyectan dentro de la luz tubárica. D) Cortes histológicos de fimbria con las proyecciones digitiformes que la caracterizan. ñas estructuras que podrían corresponder a un útero, trompa y ligamento redondo y orificio inguinal profundo permeable (Figuras 1 y 2). Del lado derecho se identificó el conducto deferente y los vasos espermáticos de características normales. Los restos fueron enviados a patología para el análisis histopatológico (Figura 3). El paciente evolucionó satisfactoriamente siendo egresado ese mismo día.

El cariotipo de sangre periférica fue de masculino con dos líneas celulares. Se realizaron 100 metafases con técnica GTG con nivel de resolución 500-600 bandas, cromosomas sexuales X-XY, siendo el resultado 45,X (67)-46, XY (33) mosaico de cromosomas sexuales. La primera línea celular presentó un número modal de 45 cromosomas con monosomía de X en $67 \%$, la segunda línea celular normal con 46 y un complemento sexo cromosómico XY en $33 \%$.

\section{DISCUSIÓN}

El término de escroto vacío se utiliza cuando a la exploración física no se identifica el testículo en la bolsa escrotal ni en el trayecto del canal inguinal o fuera del mismo. En estos casos el ultrasonido es de poca ayuda, siendo la laparoscopía el método más útil para establecer el diagnóstico definitivo que puede corresponder a testículos de localización intraabdominal, atrofia testicular y agenesia testicular o testículo evanescente.

Los derivados del conducto de Müller están presentes en un feto masculino hasta la octava semana de gestación, y su regresión está mediada por el factor inhibidor de Müller (FIM) producido por las células de Sertoli. El único resto de Müller en un varón normal es el utrículo prostático.

No se conoce la causa exacta del SPCM; sin embargo, se cree que es el resultado del defecto de la síntesis o liberación del FIM, o bien, de un defecto del receptor FIM en el feto masculino. El FIM es liberado por las células de Sertoli en el tejido fetal desde las siete semanas de gestación en adelante, y es responsable de la regresión del conducto de Müller. Los defectos en el gen FIM conducen a la persistencia de un útero y una trompa de Falopio en los hombres. ${ }^{10-12}$

Clínicamente, los casos de SPCM se dividen en cuatro categorías (Tabla 1). Nuestro caso estaría en un cuarto grupo de niños con escroto vacío unilateral y testículo normal contralateral, lo cual es lo más infrecuente. 


\section{REFERENCIAS}

1. Budianto IR, Tan HL, Kinoshita Y, Tamba RP, Leiri S, Taguchi T. Role of laparoscopy and ultrasound in the management of "impalpable testis" in children. Asian J Surg. 2014; 37(4): 200-204.

2. Riquelme M, Landa S, Miguel D, Meneses H, Andraca R. Laparoscopic orchidopexy in palpable and nonpalpable undescended testis. Rev Mex Urol. 2009; 69(5): 215-218.

3. López P, Ángel L, Rodríguez J, Reyes D, Escala J, Retamal M, et al. Abordaje laparoscópico en el testículo no palpable. Rev Chil Pediatr. 2009; 80 (3): 225-230.

4. Nieto J, Ordorica RM. Orquidopexia laparoscópica en testículo no descendido de localización intra-abdominal. Rev Mex Cir Endoscop. 2001; 2(4): 180-184.

5. García M, Somoza I, Lema A, Molina ME, Gómez J, Tellado M et al. Testes no palpables: ¿abordaje laparoscópico o inguinal? Cir Pediatr. 2009; 22: 69-71.

6. Castillo-Ortiz J, Muñiz-Colon L, Escudero K, Perez-Brayfield M. Laparoscopy in the surgical management of the non-palpable testis. Front Pediatr. 2014; 2: 1-4.
7. Tsujihata M, Miyake O, Yoshimura K, Kakimoto K, Matsumiya $\mathrm{K}$, Takahara $\mathrm{S}$ et al. Laparoscopic diagnosis and treatment of nonpalpable testis. Int J Urol. 2001; 8 (12): 692-696.

8. Moriya K, Morita K, Mitsui T, Kitta T, Nakamura M, Kon M et al. Impact of laparoscopy for diagnosis and treatment in patients with disorders of sex development. J Pediatr Urol. 2014; 10 (5): 955-061.

9. Escarcega P, Hernandez G, Huerta E, Lezama N, Hernandez $\mathrm{S}$, Kuri $\mathrm{G}$ et al. Orchiopexy-laparoscopy or traditional surgical technique in patients with an undescended palpable testicle. $J$ Laparoendosc Adv Surg Tech A. 2011; 21 (2): 185-187.

10. Gujar N, Choudhari R, Choudhari G, Bagali N, Mane H, Awati $\mathrm{J}$, Balachandran V. Male form of persistent Mullerian duct syndrome type I (hernia uteri inguinalis) presenting as an obstructed inguinal hernia: a case report. J Med Case Rep. 2011; 20: 5586-5591.

11. Agrawal A, Kataria R. Persistent Müllerian duct syndrome (PMDS): a rare anomaly the general surgeon must know about. Indian $\mathrm{J}$ Surg. 2015; 77 (3): 217-221.

12. El-Sherbiny M. Disorders of sexual differentiation: I. Genetics and pathology. Arab J Urol. 2013; 11 (1): 19-26. 